\title{
Serum Levels of Tumor Necrosis Factor-Like Weak Inducer of Apoptosis (TWEAK) in Patients with Severe Psoriasis Vulgaris
}

F.M Elesawy ${ }^{1}$, R.M Salem ${ }^{1}$, A.A Elfallah ${ }^{2}$ and A.A Sarhan

${ }^{1}$ Dermatology and Andrology Dept., Faculty of Medicine, Benha Univ., Benha, Egypt

${ }^{2}$ Clinical Pathology Dept., Faculty of Medicine, Benha Univ., Benha, Egypt

\author{
E-Mail: Sarhan2356@gmail.com
}

\begin{abstract}
Tumour necrosis factor (TNF)-like weak inducer of apoptosis (TWEAK) has been implicated in the pathogenesis of a variety of inflammatory disorders and autoimmune diseases. However, studies conducted on the relationship of TWEAK and psoriasis patients are limited. In this study, we aimed to explore the serum levels of TWEAK and detect the association betwen TWEAK levels associated and clinical variables.The severity of psoriasis was assessed by the Psoriasis Area and Severity Index (PASI). This study aims to evaluate the serum levels of TWEAK in severe psoriasis vulgaris patients. this study included 52 individuals, 28 males and 24 females, allocated from dermatology outpatient clinic at Benha University hospital. Participants were divided into patients group and a control group of normal individuals. All participants were subjected to complete history taking, clinical examination and laboratory assessment of TWEAK levels. The study group included 22 severe psoriasis patients, their ages ranged from 27.5 to 42.5 years old. Control group included 30 individuals, their ages ranged from 25 to 49 years old. PASI score for psoriasis cases ranged from 16-38. TWEAK levels were 5.6 \pm 1.3 $(\mathrm{ng} / \mathrm{mL})$ and $4.7 \pm 0.7(\mathrm{ng} / \mathrm{mL})$ for control group and cases group respectively without any statistical significance ( $\mathrm{p}>0.05)$. TWEAK levels are insignificantly decreased in patients with severe psoriasis vulgaris.
\end{abstract}

Keywords : Psoriasis, TWEAK, Tumor necrosis factor like weak inducer of apoptosis.

\section{Introduction}

Psoriasis is a common chronic inflammatory disease of the skin and joints affecting approximately $2-3 \%$ of the world's population. Skin lesions are characterized by red, scaly and well demarcated plaques that are the result of increased keratinocyte proliferation, dermal angiogenesis and infiltration of $\mathrm{T}$ cells, dendritic cells and monocytes [1].

Although the pathogenesis of psoriasis is still not fully understood, various proinflammatory cytokines (e.g., Tumor Necrotic Factor- $\alpha$, IL-1, IL-6, IL-17, IL-22, IL-23), and a number of cytokine receptors have been implicated in its pathogenesis and some of these are now under investigation as therapeutic targets [6].

Tumour necrosis factor (TNF)-like weak inducer of apoptosis (TWEAK) is a relatively new member of the TNF superfamily and has some pleiotropic effects, including stimulation of cell growth, apoptosis, angiogenesis and the modulation of immune responses [10].

TWEAK may play a crucial role in the pathogenesis of a variety of inflammatory disorders and autoimmune diseases such as lupus, rheumatoid arthritis and multiple sclerosis. Additionally, it has been offered that TWEAK blockage could be of therapeutic benefit in inflammatory conditions [4]. Studies regarding serum levels of TWEAK in patients with psoriasis are limited and results are variable [5].This study aimed to evaluate the serum levels of TWEAK in severe psoriasis vulgaris patients.

\section{Subjects and methods}

This comparative cross sectional study included two groups; Group A: 22 patients suffering from severe psoriasis vulgaris. Group B: 30 normal person not suffering from psoriasis and have no history of the disease as a control group.
All participants were selected from the outpatient clinic of Dermatology and Andrology Department of Benha University Hospitals. Informed consent was obtained from all participants. The study was approved by the local ethics committee on research involving human subjects of Benha Faculty of Medicine.

\subsection{Inclusion criteria}

All patients were enrolled in the study had severe plaque psoriasis according to the PASI score.

\subsection{Exclusion criteria} had:

Any subjects were excluded from the study if he/she

- Other forms of psoriasis.

- Concurrent significant medical conditions.

- Ongoing antipsoriatic therapy.

- Each patient were subjected to the following:

\subsection{Full history taking}

Including personal history, family history, psoriasis history as well as history of other skin diseases or drug intake.

\subsection{Clinical examination}

All patients will be subjected to the following :

\section{Complete general examination}

Including the body mass index.

\section{Complete cutaneous examination}

To evaluate the clinical type and severity of psoriasis using PASI score. 


\section{Laboratory investigation}

To evaluate serum TWEAK levels in severe psoriasis patients using ELISA technique.

\subsection{Blood sampling}

Five millimeters of venous blood sample were taken from each participant under complete aseptic condition , and put in a serum separating tubes and were allowed to clot then centrifuged. The resultant serum will be stored in aloquoit at $20 \mathrm{c}$ till assay.

\subsection{Statistical analysis}

The collected data was revised, coded, tabulated and introduced to a PC using Statistical package for Social
Science (IBM Corp. Released 2017. IBM SPSS Statistics for Windows, Version 25.0. Armonk, NY: IBM Corp.). Data were presented and suitable analysis was done according to the type of data obtained for each parameter.

\section{Results}

The study group included 22 severe psoriasis patients, their ages ranged from 27.5 to 42.5 years old. Control group included 30 individuals, their ages ranged from 25 to 49 years old. PASI score for psoriasis cases ranged from 16-38. All patients has severe psoriasis vulgaris Fig $(1,2)$.

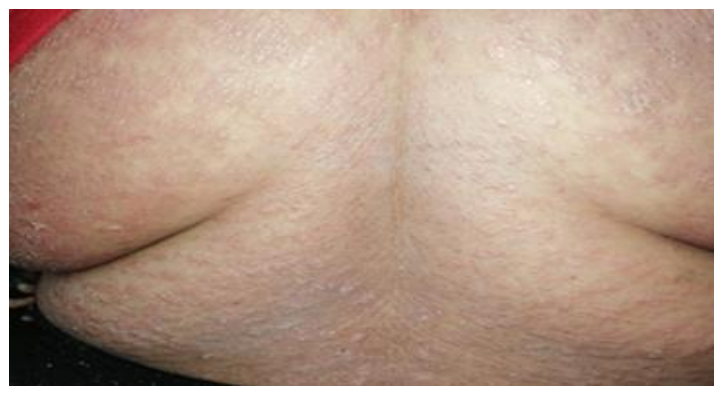

Fig (2)another patient with severe psoriasis

Fig (1) participant with severe psoriasis

The results of history taking and clinical examination are displayed in Table (1).

Table (1) Baseline clinical data in all studied Psoriasis cases.

\begin{tabular}{|c|c|c|c|}
\hline \multicolumn{3}{|c|}{ The variables } & \multirow{2}{*}{$\begin{array}{c}\text { Patients I } \\
\mathbf{N = 2 2} \\
6(27 \%)\end{array}$} \\
\hline Positive & history & $\mathrm{N}, \%$ & \\
\hline Age of o & ars) & Range & 0.1666 year -40 years \\
\hline \multicolumn{2}{|c|}{ Disease duration (years) } & Range & $10-60$ \\
\hline \multirow[t]{2}{*}{ Course } & Stationary & $\mathrm{N}, \%$ & $11(50 \%)$ \\
\hline & Progressive & $\mathrm{N}, \%$ & $11(50 \%)$ \\
\hline PASI & & Range & $16.2-38.6$ \\
\hline
\end{tabular}
(3).

There was insignificant difference between patients and control subjects regarding serum TWEAK levels ( $p>0.05)$ Fig

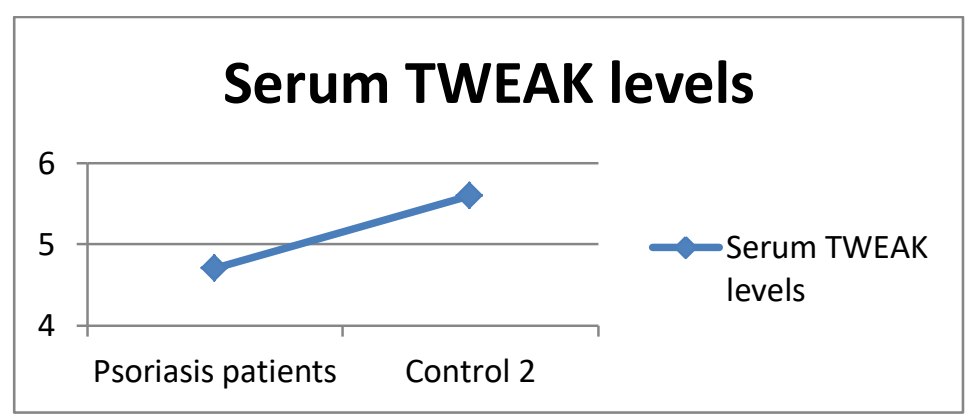

Fig (3) Serum Tweak Levels

\begin{abstract}
4. Discussion
Psoriasis is a chronic inflammatory skin disease with a strong genetic predisposition and autoimmune pathogenic traits [11)]. The classical clinical
\end{abstract}

manifestations are sharply demarcated, erythematous, pruritic plaques covered in silvery scales. Common locations include the trunk, the extensor surfaces of the limbs, and the scalp [7]. 
The hallmark of psoriasis is sustained inflammation that leads to uncontrolled keratinocyte proliferation and dysfunctional differentiation. The pathogenesis of psoriasis can be conceptualized into an initiation phase possibly triggered by trauma (Koebner phenomenon), infection, or drugs and a maintenance phase characterized by a chronic clinical picture [3].

Tumor necrosis factor-like weak inducer of apoptosis (TWEAK) has been shown to regulate a number of biological processes including cell survival, proliferation, angiogenesis, migration, and apoptosis [13].

Although TWEAK levels have been evaluated in various inflammatory and autoimmune diseases, limited studies about serum TWEAK levels in psoriasis patients have been reported.

In the present study, insignificant difference was found regarding TWEAK level between severe psoriasis and healthy control groups ( $>0.05)$. Our result were in agreement with (8) who showed that there were a nonsignificant differences in TWEAK serum concentrations between the patients and the healthy subjects.

In disagreement with the previously mentioned studies, H. Myśliwiec [9] showed that the serum TWEAK levels were significantly increased in plaque psoriasis patients when compared with the control subjects' levels.After topical treatment of exacerbated plaque psoriasis with dithranol, serum TWEAK concentrations were unexpectedly significantly elevated. This unexpected finding could be a result of both dithranol treatment and the induction of keratinocyte apoptosis [9].

Treatment with NB-UVB caused a significant reduction in PASI scores and concurrent increase in serum TWEAK. This finding may be due to increased expression of TWEAK receptor in psoriatic skin, which has been reported previously, with consequent binding of excess soluble TWEAK during treatment and subsequent release after treatment. These findings suggest a potential role of TWEAK in healing of psoriatic lesions after therapy, possibly by inducing apoptosis [9]. NB-UVB phototherapy has found also to increase TNF- $\alpha$ serum level because of an enhanced release of TNF- $\alpha$ from the exposed skin, both psoriatic and healthy [12].

As discussed above, there is a great debate about TWEAK levels in psoriasis patients either in serum or in tissue, in addition, its behavior in response to different ant psoriatic therapeutic modalities is also variable. In the era of biologic therapy and targeting specific molecules in order to increase the efficacy and minimize the adverse effects it is important to understand the role of each molecule in the pathogenesis of the disease to determine whether to antagonize or enhance it.

\section{Disclosure \\ Financial support}

No financial support was received for submission.

\section{Conflict of interest}

The authors have no conflict of interest to declare.

\section{References}

[1] J. Baliwag, D.H. Barnes, A. Johnston, Cytokines in psoriasis, Cytokine ,Vol.73, pp. 342-350, 2016.

[2] Ö. Bilgiç, A. Sivrikaya, A. Toker, Serum levels of TWEAK in patients with psoriasis vulgaris. Cytokine, Vol.77, pp.10-13,2017.

[3] W.H.Boehncke, Etiology and Pathogenesis of Psoriasis. Rheum Dis Clin North Am. 2015 Nov, Vol.41 (4), pp.66575,2015 .

[4] L.C. Burkly , J.S. Michaelson, K. Hahm, JTWEAKing tissue remodeling by a multifunctional cytokine: role of TWEAK/Fn14 pathway in health and disease, Vol.40 , pp.1$16,2014$.

[5] T. Chen, Z.P.Guo, M.M. Li, (Tumour necrosis factor-like weak inducer of apoptosis (TWEAK), an important mediator of endothelial inflammation, is associated with the pathogenesis of HenochSchonlein purpura,Clin. Exp.Immunol, vol .166 , pp.64-71,2011.

[6] A. Dowlatshahi, E.A. Evan der Voort, L.R. Arends, Markers of systemic inflammation in psoriasis: a systematic review and meta-analysis, Br.J. Dermatol, Vol.169, pp. 266-282,2013.

[7] W. Masson, M. Lobo, G. Molinero, Should all patients with psoriasis receive statins? Analysis according to different strategies. Anais Brasileiros de Dermatologia , Vol. 94(6), pp.691-697,2019.

[8] H. Myśliwiec, P. Kiluk, P. Myśliwiec, Influence of narrowband ultraviolet B phototherapy on serum tumour necrosis factor-like weak inducer of apoptosis (TWEAK) in patients with psoriasis. Clinical and Experimental Dermatology,Vol.42(7), pp.786-790,2017.

[9] H. Myśliwiec, P. Myśliwiec, A. Baran, Dithranol treatment of plaque-type psoriasis increases serum TNF-like weak inducer of apoptosis (TWEAK). Advances in Medical Sciences, vol,61(2),pp. 207211,2016

[10] S. Peternel, T. Manestar-Blaz ic', I. Brajac, Expression of TWEAK in normal human skin, dermatitis and epidermal neoplasms:association with proliferation and differentiation of keratinocytes, J. Cutan. Pathol, Vol. 38 , pp.780789,2011.

[11] A. Rendon, K. Schäkel, Psoriasis Pathogenesis and Treatment. International journal of molecular sciences, Vol. 20(6), pp.1475, 2019.

[12] Marta , Sottini, Alessandra, Serum levels of tumor necrosis factor-alpha in patients with psoriasis before, during and after narrow-band UVB phototherapy ,Vol. 50(10), pp.223-232, 2017

[13] S. Wang, Z. Xie , Z. Shen, Serum procalcitonin and $C$-reactive protein in the evaluation of bacterial infection in generalized pustular psoriasis. Anais Brasileiros de Dermatologia , Vol. 94(5), pp.542548,2016 
